Referencia para citar este artículo: Victoria-Morales, M. I., Hernandez-Wolfe, P., Acevedo-Velasco, V. E. \& GonzálezVictoria, A. (2017). Saberes e interculturalidad: Dilemas y aprendizajes en una experiencia con afrodescendientes colombianas. Revista Latinoamericana de Ciencias Sociales, Niñez y Juventud, 15(1), pp. 519-531.

\title{
Saberes e interculturalidad: Dilemas y aprendizajes en una experiencia con afrodescendientes colombianas*
}

\author{
MARÍA IRENE VICTORIA-MoRALES ${ }^{* *}$ \\ Profesora-investigadora Pontificia Universidad Javeriana Cali, Colombia. \\ PILAR Hernandez-WolFE*** \\ Profesora-Investigadora Universidad Lewis \& Clark, Estados Unidos. \\ VICTORIA EUGENIA ACEVEDo-VELASCO ${ }^{* * * *}$ \\ Profesora-Investigadora Pontificia Universidad Javeriana Cali, Colombia. \\ ADRIANA GONZÁLEZ-VICTORIA ${ }^{* * * * *}$ \\ Investigadora independiente, Colombia.
}

\section{Artículo recibido en marzo 2 de 2016; artículo aceptado en mayo 6 de 2016 (Eds.)}

- Resumen (analítico): Este artículo describe los resultados de la investigación: Diálogos afrocéntricos y occidentales en el entrenamiento de terapeutas comunitarios, que se desarrolló en 2013 con una metodología de acción participativa. El propósito del estudio fue analizar cómo un grupo de mujeres colombianas afrodescendientes, desplazadas de la Costa Pacifica en la ciudad de Cali, construyeron sentido en un encuentro pedagógico con un grupo de estudiantes estadounidenses del programa de terapia familiar de la universidad Lewis \& Clark, en Portland, Oregon. Los resultados examinan los siguientes temas: el proceso de las mujeres de construcción colectiva de sentido sobre sus saberes como prácticas de resistencia; el significado crítico, nuevo y emergente de las prácticas culturales como una posibilidad de reconfigurar la identidad colectiva; la reconstrucción del tejido social; y el fortalecimiento de las eficacias simbólicas al trabajar terapéuticamente con familias.

Palabras clave: Investigación Participativa, comunicación intercultural, interacción cultural, acción cultural (Tesauro de Ciencias Sociales de la Unesco).

Este artículo de investigación cientifica y tecnológica se deriva de la investigación denominada: "Colaboración y equidad en el entrenamiento de terapeutas familiares comunitarios: Diálogo de saberes afro céntricos y occidentales" aprobada y financiada por la Pontificia Universidad Javeriana y Lewis \& Clark College, Portland, Oregon, el 22 de marzo de 2013 con el ID 00003914, en el marco de la Política de Internacionalización de la PUJ. Inicio 23 de abril de 2013, finalización 20 de mayo de 2015. La metodología que utilizamos tiene fundamento en la Investigación Acción Participante. Área de conocimiento: Sociología. Sub-área de conocimiento: Estudios de la Familia y Estudios de Género.

** Antropóloga. Universidad del Cauca, Magistra en Psicología. Universidad del Valle. Miembro del grupo de investigación. Estudios en Cultura, Niñez y Familia. Pontificia Universidad Javeriana. Profesora Asociada, Maestría en Familia, Pontificia Universidad Javeriana, Cali-Colombia, Suramérica. Correo electrónico: ivictoria@javerianacali.edu.co

**** Psicóloga. Miembro internacional del grupo de investigación Estudios en Cultura Niñez y Familia. Pontificia Universidad Javeriana Cali. Associate Professor, Marriage, Couple \& Family Therapy Program, Lewis \& Clark Graduate School of Education and Counseling, 615 SW Palatine Hill Rd MSC 86, Portland, Oregon 97219. Correo electrónico: pilarhw@1clark.edu.co

**** Psicóloga. Miembro del grupo de investigación Estudios en Cultura, Niñez y Familia, Pontificia Universidad Javeriana. Profesora Titular, Maestría en Familia, Pontificia Universidad Javeriana, Cali-Colombia, Suramérica. Correo electrónico: veacevedo@javerianacali.edu.co

***** Comunicadora Social. Universidad del Valle. Candidata a Magíster en Comunicación Audiovisual. Pontificia Universidad Católica de Argentina. Investigadora independiente. Correo electrónico: gonzalezvictoriaadriana@yahoo.com 


\section{Dialogue of knowledges and interculturality: Dilemmas and learnings from an experience with afro-colombian women}

- Abstract (analytical): This paper describes the results of the study titled "Afrocentric and Western dialogues: Towards training community family therapists. This study was conducted in 2013 using a Participatory Action Research methodology. The purpose of the study was to analyze how displaced Afro-colombian women who were living in the Pacific Coast and were internally displaced to the city of Cali constructed meaning as part of a pedagogical encounter with a group of master's students studying Family Therapy from Lewis \& Clark College, Portland, Oregon. The results examine the following themes: the women's collective constructing meaning for their own knowledge as a form of practicing resistance; critical, new and emerging meaning from cultural practices that represent a possibility of reconfiguring collective identity; reconstruction of social fabric; and the strengthening of symbolic actions when engaging in therapeutic work with families.

Key words: Participatory research, intercultural communication, cultural interaction, cultural action (Social Science Unesco Thesaurus).

\section{Diálogo de Saberes e Interculturalidade: Dilemas e aprendizagens em uma experiência com mulheres afrodescendentes colombianas}

- Resumo (analítico): Neste artigo apresentamos os resultados da pesquisa: Diálogos afrocêntricos e ocidentais no treinamento de terapeutas comunitários, desenvolvida no ano 2013 mediante a metodologia de ação participativa. O propósito do estudo foi analisar como um grupo de mulheres colombianas afrodescendentes, deslocadas da Costa Pacifica na cidade de Cali, construiu sentido em um encontro pedagógico com um grupo de estudantes estadunidenses do programa de terapia familiar da Universidade Lewis \& Clark, em Portland, Oregon. Os resultados examinam os seguintes temas: o processo das mulheres de construção coletiva de sentido sobre seus saberes como práticas de resistência; significado crítico, novo e emergente das práticas culturais como uma possibilidade de reconfigurar a identidade coletiva; reconstrução do tecido social; e o fortalecimento das eficácias simbólicas ao trabalhar terapeuticamente com famílias.

Palavras-chave: Pesquisa participativa, comunicação intercultural, interação cultural, ação cultural (Thesaurus de Ciências Sociais da Unesco).

\section{-1. Introducción. -2. Método. -3. Resultados. -4. Conclusiones. -Lista de referencias.}

\section{Introducción}

Las experiencias interculturales de formación ofrecen oportunidades de aprendizaje transformadoras entre interlocutores que generalmente tienen remotas posibilidades de tener contacto significativo. Estas experiencias pueden incluir desafíos a las formas habituales de aprendizaje, a la investigación en contextos que presentan retos a la equidad, al pensamiento crítico y a la aplicación del conocimiento. Cuando los agentes educadores prestan atención a los problemas globales y locales de la equidad, de la historia y del posicionamiento social desde una perspectiva de aprendizaje colaborativo, se generan profundas lecciones en relación con el patrimonio cultural y con la humildad, la reflexividad y la capacidad de respuesta colaborativa. Las experiencias de aprendizaje intercultural también tienen el potencial de descentrar el privilegio y cuestionar el predominio de culturas dominantes. El propósito de esta experiencia de inmersión cuyos participantes fueron un grupo de mujeres colombianas afrodescendientes y un grupo de estudiantes de maestría en terapia familiar estadounidenses, fue el de generar un espacio de aprendizaje colaborativo que asumiese de frente los retos de la equidad en la interlocución y la pedagogía entre interlocutores con diversidades culturales, lingüísticas, de clase, raza y educación formal. 
Este artículo aborda la experiencia de aprendizaje de las mujeres afrodescendientes desarrollada a partir de este diálogo de saberes; las experiencias de los estudiantes y las estudiantes fueron examinadas previamente por Hernandez-Wolfe, Acevedo-Velasco, Victoria y Volkmann (2015). Presentamos los resultados del análisis cualitativo de los grupos focales realizados con ellas después del encuentro con el grupo de estudiantes. El conocimiento generado por estos grupos evolucionó a lo largo de las experiencias de aprendizaje planeadas con anticipación por las tres primeras autoras, situadas en un contexto de aprendizaje intercultural y transnacional. La interpretación de la experiencia pedagógica privilegia la lente de las tres primeras autoras que planearon e implementaron este programa de intercambio pedagógico ofreciendo una perspectiva crítica del proceso.

\section{- Planeación de la experiencia pedagógica colaborativa}

El programa de maestría en familia de la Pontificia Universidad Javeriana de Cali (PUJ) y el programa de maestría en terapia familiar de la Universidad Lewis \& Clark (L\&CC) de Portland, Oregón, acordaron realizar este proyecto educativo teniendo en cuenta cómo la equidad cultural, las relaciones de poder y la humildad cultural, juegan un papel central en un encuentro pedagógico internacional, como características únicas relativas a los sujetos interlocutores de la experiencia (Helms, Nicholas \& Green, 2010, Hernandez-Wolfe, 2013, Victoria-Morales, Hernandez-Wolfe \& Acevedo-Velasco, 2013). Formulamos el proceso a la luz de la siguiente pregunta: ¿de qué maneras se puede generar equidad en una relación colaborativa entre estos dos grupos para propiciar complementariedades epistémicas, enriqueciendo la creación de renovadas maneras incluyentes de comprender e intervenir las realidades sociales en una productiva tensión relacional? Para responder esta pregunta, utilizamos los planteamientos del proyecto colonialidad/modernidad (Dussel, 2005, Escobar, 2003, Mignolo, 2000, Walsh,
2006) y el enfoque de la investigación acción participativa propuesto por Borda (1986).

El proyecto denominado Modernidad/ Colonialidad/Decolonialidad (MCD), asume que colonialidad y modernidad son dos caras de la misma moneda, que opera desde el siglo XVI, cuando se inaugura la expansión colonial europea, sólo que la lógica de la modernidad ha encubierto la lógica de la colonialidad. Estos postulados teóricos sirven para proveer un marco interpretativo sobre la situación de subordinación que experimentan los pueblos indígenas, al imponerse la gramática cultural dominante que les ha negado el poder, el conocimiento y la subjetividad. Además, la perspectiva decolonial no pretende negar o ignorar la modernidad en el ejercicio a la crítica colonial. Desde esta perspectiva, se concibe a los pueblos indígenas como portadores de un paradigma epistemológico silenciado $\mathrm{y}$ negado por la "gramática" cultural dominante (Mignolo, 2012).

Según Quijano (2000), la colonialidad del poder se instala en el origen mismo del colonialismo europeo en "América Latina" con el inicio de la colonización. Los pueblos indígenas y los esclavos y esclavas africanos fueron las primeras entidades colonizadas en todos los sentidos de la palabra dominación, y el comienzo de la jerarquización de las culturas, donde el patrón de poder de la cultura dominante atraviesa todos los ámbitos de la vida social, política, económica, epistemológica, racial, sexual y subjetiva. Para Quijano (2000), la colonialidad del poder no sólo consistió en ponerle nombre a los sujetos, sino implicó un proceso para justificar la imposición de una "gramática cultural" que se institucionalizó en la práctica cotidiana desde el momento mismo de la colonización. Walsh (2006), MaldonadoTorres (2003) y Albán (2006), han argumentado de manera crítica, en el contexto de los estudios decoloniales, la colonización del conocimiento y la colonización del ser. Al posicionamiento privilegiado del conocimiento disciplinado occidental, legitimado para normar, teorizar y universalizar una visión categorial hegemónica del mundo, le antecede una larga historia de consolidación de los idearios de la modernidad. La visión teleológica de la historia y la razón 


\section{María Irene Victoria-Morales - PILAR Hernandez-Wolfe - \\ VICTORIa EUGENIA ACEVEDO-VELASCO - AdRIANA GONZÁlEZ-VICTORIA}

guiada por los cánones del positivismo legitimadores del conocimiento como objetivo y verdadero, desplazó e invisibilizó la pluralidad de maneras de ser, estar y producir conocimiento en la praxis social por fuera de los cánones establecidos para hacer ciencia. Es justamente en este avasallamiento y negación donde reside el acto de colonialidad del conocimiento.

La colonialidad del conocimiento y la colonialidad del ser forman una diada indisoluble, constituyéndose en requisito de existencia mutua; una de las invenciones de la modernidad soportada en una taxonomía de la humanidad, marcó el encuentro con la alteridad, con "otro" colonizado; Europa fue clasificada en la teoría del evolucionismo social como modelo civilizatorio, y los pueblos colonizados, disminuidos en su humanidad, racializados e interiorizados -cercanos a la animalidadcomo antítesis del modelo europeizante, creándose así el espectro interpretativo para generar la colonialidad del ser vivida hasta el momento contemporáneo (Albán, 2006, Maldonado-Torres, 2003, Victoria-Morales, 2011). Walsh (2006) plantea la colonialidad del ser como negación de la condición humana y objetivación-cosificación de la vida de los sujetos subalternos, los negros y negras, los indios e indias, los individuos desposeídos, y esta objetivación tiene implícita la negación ontológica de los conocimientos que orientan el vivir de los pueblos y que dan sentido a su existencia. Albán (2006) reconoce que, en el camino de la decolonización, la interculturalidad como proyecto epistémico y político propone el diálogo con el reposicionamiento de los saberes locales o propios en las coordenadas de poder regulatorias de la producción y legitimación del conocimiento, así como la deconstrucción del sistema clasificatorio basado en la raza, el género y la clase, dispositivos en los cuales se sustenta la colonialidad del ser.

Una pedagogía decolonial (Walsh, 2007) requiere de procesos permanentes de resignificación para problematizar los relatos de la colonialidad que producen subjetividades inscritas en las oposiciones binarias: superiorinferior; de igual manera, para construir nuevos posicionamientos y sentidos a los saberes locales y a las historias subalternas, reconociéndolos

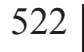

como potenciales de trasformación social, sin que transiten en la institucionalidad legitimadora del conocimiento. Así, resignificar es una acción potente que, desde nuestra perspectiva, es fundamental en la búsqueda de caminos de decolonialidad. En su dimensión pragmática requiere de la intersubjetividad para desatar ejercicios deliberativos centrados en la argumentación y la interrogación, para erosionar los efectos de la colonialidad en las subjetividades e identidades colectivas.

Este proyecto pedagógico compartió las intencionalidades de la interculturalidad, y privilegióladisrupciónal propiciarel aprendizaje mutuo entre dos actores posicionados en lugares epistémicos asimétricos: Por un lado, las mujeres afrodescendientes llamadas maes $^{1}$, hacedoras de la escuela Sé Quién Soy, cuyo trabajo gira en torno a la recuperación y preservación de los saberes propios involucrando a las familias de la costa Pacífica colombiana desplazadas por la violencia del conflicto armado. Por otra parte, un grupo de profesionales estudiantes $-\mathrm{PE}^{2}$ - de la maestría en Terapia Familiar y de Pareja de L\&CC. Para ellos y ellas la experiencia se estructuró como parte de un curso electivo dentro del énfasis en Terapia Familiar Internacional, orientado por una de las autoras (PHW) y cuyo contenido temático discutía asuntos de colonización y decolonización, consistentes con las visiones de autores y autoras de Latinoamérica y de movimientos sociales (Escobar, 2010, Freire, 1972, Hernandez-Wolfe, 2013).

El contacto y la cooperación de los estudiantes y las estudiantes con las mujeres afrocolombianas y con los profesores y profesoras locales, comenzaron en el curso prerequisito previo a la experiencia de inmersión a través de reuniones por Skype, donde se conocieron y generaron lluvias de ideas sobre los temas que querían aprender acerca de cada quien. Cada grupo discutió temas para confrontar, en un formato de taller durante

\footnotetext{
1 La palabra mae en la costa Pacífica designa a la madre, y en el contexto de la escuela las mujeres la retoman para nombrarse como mamás maestras. En adelante en este artículo nos referiremos a ellas con este vocablo, maes.

2 Grupo conformado por once profesionales psicólogos y psicólogas. En adelante los nombraremos con la sigla PE.
} 
la visita. Las mujeres afrodescendientes se reunieron con otra autora (IV) para discutir sus necesidades y planificar sus talleres para los estudiantes y las estudiantes. Las mujeres sugirieron la equidad de género, la sexualidad y el liderazgo como temas para talleres que querían recibir. Los estudiantes y las estudiantes seleccionaron temas como los rituales relativos al nacimiento de un niño, la pérdida y el duelo, la gastronomía afrocolombiana y el sentido de comunidad, así como las tradiciones orales que implican la recitación y el canto de décimas, coplas y otras formas musicales utilizadas en estas comunidades para mantener la identidad de los pueblos afrocolombianos de la costa del Pacífico.

\section{Método}

\section{Participantes}

Esta experiencia incluyó a tres mujeres heterosexuales, profesoras colombianas (psicología y antropología), dos de ellas vinculadas a la PUJ y una de ellas a L\&CC, y ocho mujeres afrodescendientes entre los 30 y los 60 años desplazadas a la ciudad de Cali por la violencia tanto de las guerrillas como de los paramilitares en el sur del país. Su nivel de educación oscilaba entre el octavo grado y la licenciatura; sus ingresos eran -o siguen siendobajos y provienen de la economía informal. Las mujeres afrocolombianas y líderes de la comunidad habían trabajado con una de las autoras (IV) durante más de quince años en diversos proyectos activistas -derecho a la vivienda e identidad cultural-. Estas mujeres desarrollaron una escuela informal local "Sé Quién Soy", para recuperar sus tradiciones e inculcar en sus hijos e hijas un fuerte sentido de la identidad cultural ante los cambios drásticos de su vida en la ciudad que poco a poco van erosionando sus creencias, sus tradiciones, su conexión con la tierra y con sus animales domésticos y la naturaleza. Esta escuela adapta su tiempo y espacio para la enseñanza según las necesidades de la comunidad, e integra tradiciones orales, musicales y educativas practicadas en las zonas rurales, donde por siglos la comunidad afrocolombiana creció y se desarrolló a pesar de la negligencia política, económica y social del Estado, y la cultura dominante. Su plan de estudios incluye una forma afrocéntrica y local de conocimientos, y proporciona la posibilidad de diálogo e intercambio de conocimientos entre ellas y el colectivo de estudiantes de terapia familiar en la formación.

El grupo de estudiantes de L\&CC incluyó ocho mujeres heterosexuales de clase mediaalta y tres hombres heterosexuales de clase media-alta. De los once individuos estudiantes, había dos latinos, una filipina-judía, una asiática de Sudáfrica, un asiático-europeo americano y seis europeos-americanos; cinco eran bilingües, cinco tenían un nivel intermedio-avanzado de español y uno tenía un nivel inicial.

\section{Procedimiento}

Lainvestigación-acción-participativa(IAP) conjuga las actividades del conocimiento de la realidad mediante mecanismos de participación de la comunidad, para el mejoramiento de sus condiciones de vida. El proceso investigativo de la IAP está basado en un sistema de discusión, indagación y análisis, en el que las personas participantes forman parte del proceso al mismo nivel que el sujeto investigador. Las teorías no se desarrollan de antemano, para ser comprobadas o esbozadas por el sujeto investigador a partir de un contacto con la realidad. La realidad se describe mediante el proceso por el cual una comunidad crea sus propias teorías y soluciones sobre sí misma (Borda, 1986). Para el análisis de datos usamos la teoría fundamentada (Glaser \& Strauss, 1967, Lincoln \& Guba, 1985, Padgett, 1998). Grabamos y transcribimos los diálogos en tres grupos focales, los que orientamos teniendo en cuenta la reflexividad dialógica propuesta por Ghiso y Tabares-Ochoa (2011), quienes reconocen "la capacidad de los sujetos para interrogar lo dicho, lo hecho y lo pensado, “... una opción metodológica que permite abordar memorias, prácticas y discursos en sus múltiples formas expresivas" (p. 129). Codificamos las transcripciones en dominios que acordamos por consenso dentro del equipo de investigadoras y líderes. El proceso de consenso involucró la revisión individual de cada 


\section{María IRENe Victoria-Morales - PILAR HerNandez-Wolfe - \\ VICTORIA EUGENIA ACEVEDO-VELASCO - ADRIANA GONZÁLEZ-VICTORIA}

transcripción, y reuniones para dialogar sobre las interpretaciones realizadas. Los métodos de triangulación, es decir, el proceso de corroborar de varias formas la interpretación de los datos en la investigación cualitativa para fortalecer su exactitud y la credibilidad (Lincoln \& Guba, 1985), se basaron en el uso de grupos focales y de resúmenes de casos, en la codificación por separado, y en discusiones para alcanzar un consenso en el proceso de codificación y de las categorías seleccionadas, así como en la inclusión de una cuarta investigadora (AG).

\section{Resultados}

El análisis de datos nos permitió identificar las siguientes temáticas: (a) Posicionamiento en el espacio dialógico; (b) afirmación de la tradición como experiencia de vida y resistencia al folklor; (c) retos en torno a las relaciones de género; (d) afirmación de los saberes propios para elaborar el duelo; y (e) el papel de cuidadoras.

\section{- Posicionamiento en el espacio dialógico}

Esta experiencia de intercambio pedagógico fue diseñada por las tres primeras autoras, de manera que hubiese un conocimiento e introducción a la cultura de cada quien, con previo examen de las diferencias de poder estructurales e interpersonales presentes en un encuentro de este tipo. Aunque estas diferencias no se pueden erradicar, las profesoras las hicieron evidentes y generaron discusiones en torno a los factores identitarios y las relaciones de poder. También diseñamos un espacio de diálogo en el cual los estudiantes hombres y mujeres, y las mujeres participantes, ofrecieron $\mathrm{y}$ decidieron en conjunto cuáles temas iban a presentar al otro para diseñar los talleres que conformarían el encuentro pedagógico de seis talleres. Cada grupo ofreció tres. La reflexión de las mujeres en torno a las decisiones y a la realización de los talleres generó dos asuntos clave: (1) un cambio en el lugar de enunciación como sujetos que tienen voz, agencia y poder de decisión, y (2) empoderamiento para pedir respuesta concreta a sus expectativas y necesidades.

En este encuentro las personas participantes estaban y están ubicadas estructuralmente en coordenadas histórico-temporalesespaciales opuestas y en tensión en el mundo representacional: el Norte desarrollado frente al Sur subdesarrollado, los blancos "gringos" frente a los negros, los saberes académicos frente a los saberes locales. En este proceso, ese "otro" significaba para ellas la oportunidad de confrontar imaginarios; de tener a un otro diametralmente diferente frente a sus ojos: "los gringos, quienes hablan otra lengua, comen otras cosas" ... El gringo adquiere la condición de un "otro exótico": "Me los imaginaba más jóvenes, blancos, que no entenderíamos lo que ellos hablaban. Al verlos me cambió toda la imagen que yo tenía; el impacto fue que en cuanto los vimos caímos en confianza".

Tomar el lugar de enunciación como maestras de los PE provocó durante el encuentro un giro en las tradicionales relaciones de poder pedagógicas enmarcadas en la unilateralidad del binarismo superior-inferior, quien enseña $\mathrm{y}$ quien aprende. Este giro fue vivenciado $\mathrm{y}$ reflexionado por las mujeres: "ahí estuvimos aportando todos, tanto ellos como nosotras; siento que hubo una igualdad, ellos en lo de ellos y nosotras en lo de nosotras, pero estuvimos iguales compartiendo saberes". No obstante, las maes debieron enfrentar muchos temores surgidos del proceso histórico de subordinación por su género y su raza:

Tuvimos muchos miedos al enfrentar el reto de enseñar a los profesionales, pero sabíamos que teníamos mucho que aportar; fue dura la tarea de ordenar nuestros pensamientos, de preguntarnos, por un lado, por nuestros saberes propios, por el papel de ellos en nuestro territorio de origen para mantenernos unidos $\mathrm{y}$, por otro, repasar las cosas que hemos hecho en la escuela Sé Quién Soy acá en la ciudad. Sentimos que pudimos armar y contar nuestra historia a nuestra manera, en oralidad $^{3}$, y allí nos dimos cuenta

3 La oralidad se concreta en décimas, poemas, cantos, cuentos y adivinanzas. Las décimas son de mayor recurrencia en cuanto a su uso. 
que hacer mayor conciencia de lo que hacemos fortaleció nuestra organización, nos acrecentó la dignidad de ser negras.

Este descubrimiento de un otro con quien confrontar los prejuicios y con quien dialogar generó confianza y deseo de compartir la riqueza cultural y pedagógica de las maes:

Bombo cununo y guasa

A la escuela resaltar

Bombo cununo y guasa esta escuela es ancestral.

Esta escuela "Sé Quién Soy “

Donde voy me reconozco

Donde voy yo soy historia

Modelo para otros.

En la escuela han recuperado y construido enriquecidas narrativas acerca de quiénes son articulando su pasado con su presente tal como se expresa en este canto:

Estas mujeres salidas de quebradas

Con los procesos se ponen a la luz

Para que ellas expongan en los grandes espacios

Con una mente libre de esclavitud

Porque de pueblos menos favorecidos

Salen mujeres dotadas de virtud.

En torno al siguiente punto: el empoderamiento para pedir respuesta concreta a sus expectativas y necesidades, las reflexiones y cambios se dieron después del primer taller que llevaron a cabo los PE. A pesar de conocer el trabajo de las maes, haber escuchado sus décimas, haber leído el trabajo del antropólogo Arturo Escobar con las comunidades negras en Colombia y haber tenido una preparación extensa en alternativas de aprendizaje e interculturalidad, ofrecieron un taller como lo hacían en sus clases de la universidad (L\&CC) con estudiantes como ellos y ellas. Es decir, con un formato que incluyó una presentación en Power Point, cátedra magistral y espacio para preguntas y respuestas. De tal modo que las maes no sólo no se motivaron a participar activamente en el taller sino que consideraron que no podían aprender de esta manera. Las mujeres afrocolombianas no suelen sentarse durante largas horas para ser enseñadas sobre un tema; tuvieron dificultades para entender el propósito de las actividades preparadas y las consideraron similares a las del entorno escolar en la que se les enseñó en una escuela tradicional. Los individuos estudiantes se sintieron decepcionados de sí mismos, pero la experiencia les obligó a cambiar y ser más eficaces en la participación, la comunicación y el trabajo con las maes. Tomaron la iniciativa de escuchar la opinión de las mujeres y volvieron a la mesa de trabajo. Se expusieron a tomar el riesgo de dejar sus formas habituales de conocer y relacionarse para conectarse con ellos y ellas en un espacio donde pueden coexistir ambos grupos. Las mujeres se expusieron en un espacio donde podían expresar opiniones, disentir, confrontar el proceso y ser escuchadas. En los siguientes talleres, los estudiantes y las estudiantes demostraron que escucharon a las maes y que podían responder a sus necesidades. Usaron medios pedagógicos que incluyeron rituales, historias y ejercicios para compartir ideas específicas sobre equidad de género, crianza y liderazgo.

\section{- Afirmación de la tradición como experiencia de vida y resistencia al folklor}

Las maes reflexionaron sobre una situación vivida antes del encuentro con los estudiantes y las estudiantes, de la siguiente manera:

Un grupo de personas que trabajan con mujeres violentadas, nos invitaron a compartir con ellas disque nuestro folklor, nunca fuimos porque sentimos que no hacemos folclor ni teatro, no representamos nada, así es nuestra manera de vivir. Todo eso nos dejó pensando cómo trabajar con los PE sin que nuestra historia contada como lo hacemos fuera vista como folklor, esta preocupación nos llevó a profundizar en el significado de nuestros saberes.

El encuentro les proporcionó el espacio y las enfrentó al reto de poner en práctica el ser maestras desde su tradición: "Ahora vamos haciendo nuevas fotografías, con el encuentro ${ }^{4}$ se nos proporcionó una lente para ver cosas que hemos vivido, como la violencia contra las mujeres niños y

$4 \quad$ Una de las problemáticas tratadas en el encuentro y previamente solicitada y concertada con las maes por los PE, giró en torno al enfoque de género. 
niñas, el machismo y hasta el racismo, pero que no hemos visto con el ojo de lo intolerable.

Las maes plantean así una forma distinta de relacionalidad con la tradición; la interrogan en sus contenidos y proponen descifrar sus sentidos con un sujeto interlocutor extranjero que está dispuesto a escuchar y a participar en un diálogo con ellas. Esta relación da paso a la posibilidad de recuperar, resignificar y poner en práctica su método para enseñar sobre sus historias. Este escenario del encuentro afianza un sentido político de su quehacer: "La escuela afianza la dignidad de los jóvenes y viejos afro estigmatizados, menospreciados tantas veces en la escuela, en las calles, en los buses, en fin, en casi toda parte". Por esto expresan de manera afirmativa el sentido de la escuela en la siguiente décima:

"La escuela Sé Quién Soy

Es una gran estrategia

Que guarda la identidad

Y el valor de nuestra etnia

Hacemos la resistencia

Por el derecho de vivir

Al saber y al pensamiento

Para poder persistir

Así nos hacemos fuertes

En el lazo de hermandad

Sin perder las relaciones

Con nuestra comunidad

El auto reconocimiento

Nos brinda la autonomía

Del saber territorial

Con toda su jerarquía.

Las maes han conferido ahora un lugar político a su oralidad; de manera específica las décimas son una mediación en los momentos de interlocución con la institucionalidad estatal o con las ONGs, sobre todo cuando ellas reconocen actuaciones de poder arbitrario en las prácticas institucionales, tales como expresiones $\mathrm{o}$ acciones de segregación y exclusión por su condición de género o de raza. En estos casos, la décima toma un carácter de ironía para denunciar el hecho maltratante, y gracias a ella la situación se hace pública y se registra en la memoria colectiva como impugnación:

Hemos aprendido a decimar para protestar y contar los malos tratos de las instituciones aquí en la ciudad, lo importante es que la protesta queda por siempre en el corazón de la comunidad nunca se olvida y esto aviva la lucha desde la dignidad. Ojalá que los PE hubieran captado por qué decimos que lo nuestro no es folclor y que decimando se gana en la lucha y se sana el corazón.

\section{- Retos en torno a las relaciones de género}

El contexto físico y social en el que se dio esta experiencia pedagógica fue en la PUJ, una institución con poder real y simbólico a la que las profesoras y estudiantes invitados estaban vinculados. Esta fue una realidad innegable. Aunque a las maes se les reconoció su trabajo pedagógico monetariamente -como se haría con cualquier profesional en el país-, éstas, en su afán de ofrecernos lo mejor de su trabajo, invitaron a un reconocido caballero poeta de su comunidad que trabajaba tiempo completo como maestro en un pueblo remoto en otro departamento. Si bien ninguna de las mujeres tenía un trabajo estable con prestaciones sociales, y él sí, ellas decidieron invitarlo a participar en dos de sus talleres cubriendo todos sus gastos y pagándole por su trabajo. El costo de esta invitación fue muy alto en relación con los ingresos del trabajo con nuestro proyecto. Cuando las profesoras se reunieron con las maes para conversar sobre el proceso y sobre esta decisión en particular, problematizamos dicho asunto. Compartieron que querían que el mejor poeta de su comunidad se presentara a esta audiencia de extranjeros; sin embargo, no había nada que él aportara que ellas no pudieran aportar: "A mí me parece que nosotras tenemos nuestras cosas montadas y no necesitamos de nadie más, además nosotras sí hablamos de nuestros problemas como mujeres y como madres y eso es lo que yo quiero compartir".

Las maes conversaron francamente sobre todo su trabajo y sobre la manera en que no valoran suficientemente su trabajo desde lo económico. Esta fue una oportunidad para abrir el tema del género y la equidad: ¿qué les hizo privilegiar la voz de él sobre la de ellas? 
Algunas de ellas respondieron:

"en la tradición el hombre va primero... pero no tiene que ser así, es que no cuestionamos", "para mí, pues está primero el reconocimiento de él pues es muy reconocido, pero yo he trabajo muy duro en la escuela para crear mi propio reconocimiento", "así hemos sido... y a veces en la casa ipasa lo mismo!".

Fue una experiencia de aprendizaje doloroso que evidenció otros temas de este grupo de mujeres, como los procesos de toma de decisiones y de liderazgo. Llegaron a un consenso al compartir la toma de decisiones de manera más democrática. Algunos de sus comentarios fueron:

"yo creo que tenemos que actuar de una manera en que todas podamos opinar. Yo no estuve de acuerdo pero yo no dije nada porque yo no soy la que manda aquí"; "aquí las cosas funcionan de manera que uno respeta a los mayores y eso nunca lo conversamos, pero yo sí quisiera poder expresar mi opinión y en este caso pues no había que tomar esa decisión de invitarlo".

\section{- Afirmación de los saberes propios para elaborar el duelo}

En este proyecto, las maes ofrecieron enseñar a los PE qué es un duelo para ellas $\mathrm{y}$ cómo se entiende y procesa el dolor en su comunidad. Teniendo en cuenta el contexto histórico reciente, se llevaron a cabo conversaciones entre maes y estudiantes sobre el impacto de la violencia:

"Mi comadre llegó del Charco destruida; su marido fue asesinado en medio de balas cruzadas del conflicto armado; en la unidad de víctimas la atendió la psicóloga dizque le dijo que tenía que ir a varias consultas para hacer el duelo. La comadre no volvió, no le entendió lo que quería decir. Las maes la acogimos, nos acompañó varias veces en el canto de las décimas... un día había hecho sus propias décimas y en ellas narró cómo le habían matado el marido; su rabia y su dolor estaban en cada entonación de la palabra".

Las maes articularon las maneras en que sus ancestros lejanos y recientes sanaban los dolores de la muerte de sus abuelos y abuelas en el territorio, y concluyeron que los rituales de los velorios y los novenarios son los lugares destinados para generar emociones colectivas:

"No lo sabíamos pero hoy entendemos que los rezos y cantos durante las nueve noches no son para el difunto. Son maneras para acompañar a la familia, a los dolientes. Nueve noches para reunirse, para estar en comunidad, la última noche es de estremecimiento para volcarse en el grito del dolor hasta perder el sentido si es preciso. Todo esto se permite y se alienta para que los dolientes se despidan entendiendo que hasta ahí se llega, que hay que poner un límite con el sentir y la expresión extrema de la tristeza, y que la vida sigue. Entonces las comadres y los compadres, los vecinos, ofrecen la ayuda y el acompañamiento para continuar, para que no mueran de pena moral con el difunto".

La reflexión que subyace en la experiencia anterior gravita en torno a la solidaridad como bien comunitario, tejedor de condiciones de seguridad emocional ante la adversidad y también frente a la construcción de redes de reconocimiento mutuo en los acontecimientos que sostienen la vida colectiva. El sentido integrador de la salud mental se reafirma en la solidaridad, sin la cual la vida espiritual y cultural de la colectividad está amenazada.

El encuentro con los PE reafirmó a las maes en la valoración de sus saberes propios como condición indispensable de la seguridad emocional, muchas veces avasallada en lourbano por la disolución de las alianzas comunitarias, por algunos enfoques disciplinares de la salud mental que borra la dimensión de lo colectivo para enfatizar sus prácticas terapéuticas sólo desde la dimensión individual:

"También hemos recuperado la manera de conciliar conflictos a través del decimeo, como lo hacían los mayores; en la décima también se saca la rabia, la ironía, también el afecto y se registra 


\section{María Irene Victoria-Morales - PILAR Hernandez-Wolfe - \\ VICTORIa EUGENIa ACEVEDO-VELASCO - AdRIANA GONZÁLEZ-VICTORIA}

en ellos los acuerdos y así se vuelven parte de la memoria colectiva; después del encuentro lo vemos como parte de nuestro hacer terapéutico".

Una deriva significativa desde este nuevo lugar de reflexión ha movilizado a las mujeres a realizar alianzas con otras organizaciones comunitarias que trabajan sobre las violencias de género, tales como la Ruta Pacífica de Mujeres ${ }^{5}$, nodo Valle. Participan de procesos de formación sobre Género y Derechos humanos ${ }^{6}$. De igual manera, han seducido la participación de varios hombres -adultos jóvenes y niños- en el proceso organizativo de la escuela.

La escuela Sé Quién Soy, en su proceso continuado de recuperación y resignificación de memoria colectiva profundamente estimulada por la experiencia del diálogo de saberes, irrumpe en nuevos escenarios que trascienden sus espacios comunitarios para dialogar con otros actores estatales ${ }^{7}$ sobre el fortalecimiento y potenciación de sus saberes como cuidadoras de sus comunidades ante las situaciones traumáticas asociadas al desplazamiento forzado, encaradas en la des-territorialización y territorialización en la ciudad, preocupaciones que atañen al tema de la salud mental.

\section{- El papel de cuidadoras}

La toma de conciencia de su lugar de praxis como cuidadoras ocurrió durante el encuentro; aprendieron de los PE la importancia del

$5 \quad$ La Ruta Pacífica de Mujeres es una red de diversas organizaciones de mujeres constituida en todo el país. Su estructura se concreta en nodos de trabajos en las diferentes regiones y se consolida en la década de los 90 para trabajar sobre los efectos de la guerra en las mujeres en el contexto del conflicto armado colombiano.

6 Las mujeres y hombres hoy participantes de la escuela "Sé Quién Soy" han realizado procesos de formación impartidos por la Alcaldía del Municipio de Cali. Actualmente, participan en el contexto de este proyecto en un proceso de formación sobre Enfoque de Género: Derechos sexuales y reproductivos, cuyas temáticas fueron concertadas con las mujeres y las investigadoras.

7 Han participado como ponentes en: El3er. Congreso Internacional de Sistemas de Salud III Conferencia Nacional de Salud Pública. Santiago de Cali. Octubre de 2014, Organizado por la Pontificia Universidad Javeriana de Cali. El encuentro internacional de salud púbica; "De la guerra a la paz: reto para la salud pública". Diciembre, 2014. Santiago de Cali. Organizado por la Secretaría de Salud Departamental del Valle. El marco del Seminario de Políticas Públicas y Socio-antropología de la Familia. Mayo 2014. Maestría en Familia. Pontificia Universidad Javeriana de Cali. acompañamiento mediado por el respeto: "nos enseñaron algo muy importante: apóyate en mí y yo me apoyo en ti, pero respetándonos; eso para nosotras como trabajadoras comunitarias fue maravilloso, es una cosa que quedó registrada en nosotras". Las maes enseñaron a los PE las ritualidades relacionadas con otras maneras de nacer, criar, amar, enfermar, sanar y morir, en las cuales se evidenciaron claves para reorientar el trabajo de las gentes profesionales en el campo de la salud mental y comunitaria, a partir del reconocimiento de los ordenamientos simbólicos que pueden salvaguardan la vida emocional de las comunidades. Finalmente, ellas así lo expresan:

"Pienso que un grupo de mujeres de ríos que a duras penas conocen la letra y la hablan, se unen y se consideran maestras en su diario vivir y en su quehacer, es un aprendizaje que ellos llevan (...) que puedan contar sobre este grupo junto, poder llegar a una ladera de los ríos en Estados Unidos y decirles júntense que hay unas mujeres juntas en Colombia que están dignificando para dignificarse ellas mismas y hacer resistencia. (...) ojalá puedan irse al pantano en alguna comunidad de Estados Unidos y decir: vamos a partir desde su saber, desde sus orígenes. Ojalá puedan aprender que con versos también se pasan las penas".

\section{Conclusiones}

El propósito de este estudio fue dar cuenta de la experiencia de aprendizaje con un grupo de mujeres afrodescendientes, desarrollada a partir de este diálogo de saberes con estudiantes extranjeros de maestría en terapia familiar. Los resultados del análisis temático indican que las condiciones generadas en esta experiencia propiciaron la posibilidad de que ellas se posicionaran en un espacio dialógico con mayor equidad, lo que facilitó el desarrollo de su agencia personal y de grupo. También afirmaron su tradición como experiencia de vida y resistieron el que se les impusiera ver su tradición como folklor; se confrontaron con sus propias maneras de subvalorarse en las relaciones de género, afirmaron sus saberes 
propios para elaborar el duelo y reafirmaron su papel de cuidadoras.

El abordaje de las narrativas del colectivo de mujeres afrodescendientes de la escuela Sé Quién Soy, generadas por el encuentro con un "otro" cuyo lugar en la oposición inferiorsuperior lo define como superior, evidencia los efectos movilizadores respecto a perspectivas críticas surgidas en las dinámicas de los diálogos de saberes realizados bajo intencionalidades disruptivas para provocar una horizontalidad dialógica trastocando las posiciones epistémicas de enunciación asimétricas de los individuos participantes. Este trastrocamiento de las posiciones epistémicas de enunciación asimétricas se logró a partir de un proceso de reconocimiento mutuo de las personas participantes sobre la valoración de sus saberes; por un lado, un saber académico relativizado en su hegemonía y, por otro, un saber propio posicionado desde su legitimidad histórica como eje de resistencia y pervivencia. La tensión entre la relativización y el reconocimiento de legitimidad estuvo mediada por el cuidado de no convertir el encuentro en una experiencia de acentuación de la colonialidad del ser; es decir, sin provocar el «exceso ontológico» que ocurre cuando seres particulares se imponen sobre otros y, más allá de esto, la efectividad potencial o actual de los discursos con los cuales el otro responde a la supresión como un resultado del encuentro (Maldonado-Torres, 2003).

Sin embargo, es necesario plantear uno de los dilemas más claros, resultante de este trabajo: ¿en qué medida la asimetría en las relaciones de poder genera sujetos dóciles que se acoplan a los deseos del interlocutor con mayor poder? ¿Es posible evitar el impacto de esta desigualdad de condiciones en la libertad que tienen los interlocutores para disentir? Para Mir y Mir (2009), la diferencia de poder que caracteriza las instituciones en los Estados Unidos y las instituciones en los "países en desarrollo" como Colombia, da lugar a la posibilidad de generar condiciones para trabajar con un participante más gobernable, es decir, menos hostil a las sugerencias procedentes de los representantes de estas universidades. Si bien la participación de las mujeres afrocolombianas se planeó cuidadosamente para que participaran activamente en un proceso de colaboración con los alumnos y alumnas de L\&CC, consideramos que la influencia de las instituciones y el país que algunos de nosotros representábamos, era inevitable, y fue determinante en su deseo de trabajar con nosotros y extenderse demasiado para ayudarnos con el proyecto. Sostenemos que esta condición motivó su decisión de invitar al caballero poeta, aunque esto les representase consecuencias económicas negativas. La antropóloga Rita Segato (2003) sostiene que históricamente la dignidad de los pueblos se cimienta en la sumisión de sus mujeres.

Es en el cuerpo femenino y en su control por parte de la comunidad que los grupos étnicos inscriben su marca de cohesión. Hay un equilibrio y una proporcionalidad entre la dignidad, la consistencia y la fuerza del grupo y la subordinación femenina (Segato, 2003, p. 10).

Esta es una subordinación de la cual no han escapado las maes; la lucha por recuperar los saberes propios ancestrales se ha venido perfilando a contracara de una exterioridad que podríamos llamar continuum de colonialidad; desde ese hacer se han reconocido como sometidos a un poder heterónomo que deja de lado la reflexión sistemática sobre sus dinámicas internas de reproducción de expresiones de poder de la misma colonialidad, presentes en las relaciones de género y generacionales que, aunque articuladas a procesos históricos estructurales, no se habían asumido como objeto de transformación inmediata en su quehacer organizativo.

El saber propio de las maes, expresado en su oralidad, revela su potencial político en la medida en que se convierte en un cohesionador organizativo, crea y recrea una historicidad de su quehacer cotidiano. Son saberes que podríamos entender -como lo plantea De Sousa (2010)- nacidos de la lucha de resistencia. Los estudios de la colonialidad instan cada vez más a la academia a consolidar prácticas pedagógicas con sentido decolonial; esto significa que la academia cuestione su lugar de privilegio para producir conocimiento sobre los otros subalternizados, y se permita interlocutar con ellos desde un posicionamiento simétrico para que realmente no sean objetos de enunciación 
sino sujetos de enunciación (Walsh, 2006).

Quizás podamos recurrir a la experiencia de la escuela Sé Quién Soy que acoge la diversidad para permanecer en la unidad. Los proyectos educativos transnacionales concebidos dentro de una epistemología iluminada por el pensamiento descolonizador, permiten la posibilidad del multilingüismo, la interculturalidad, y pedagogías alternativas. Cuando aquellos y aquellas cuya responsabilidad es enseñar tienen en cuenta los dilemas e implicaciones de la falta de equidad, es posible generar espacios que desestabilicen el predominio del pensamiento eurocéntrico, dando paso a la articulación y aprendizaje de valiosos conocimientos locales.

\section{Lista de referencias}

Albán, A. (2006). Conocimiento y lugar: Más allá de la razón hay un mundo de colores. Texiendo textos y saberes. Cinco hilos para pensar los estudios culturales, la colonialidad y la interculturalidad, (pp. 59-82). Popayán: Universidad del Cauca.

Borda, O. F. (1986). El problema de cómo investigar la realidad para transformarla. Bogotá, D. C.: Tercer Mundo.

De Sousa, B. (2010). Descolonizar el saber, reinventar el poder. Montevideo: Trilce.

Dussel, E. (2005). Europa, modernidad y eurocentrismo. En E. Lander (comp.) La colonialidad del saber: Eurocentrismo $y$ ciencias sociales. Perspectivas Latinoamericanas, (pp. 41-56). La Habana: Editorial de Ciencias Sociales.

Escobar, A. (2003). Mundos y conocimientos de otro modo. El programa de investigación de modernidad/colonialidad latinoamericano. Tabula Rasa, (1), pp. 51-86.

Escobar, A. (2010). Territorios de la Diferencia: Lugar, Movimiento, Vida, Redes. Popayán: Samava Impresiones.

Freire, P. (1972). El mensaje de Paulo Freire. Teoría y práctica de la liberación. Madrid: Inodep.

Ghiso, A. \& Tabares-Ochoa, C. M. (2011). Reflexividad dialógica en el estudio de jóvenes y prácticas políticas. Revista Latinoamericana de Ciencias Sociales, Niñez y Juventud, 9 (1), pp. 129-140.
Glaser, B. G. \& Strauss, A. L. (1967). The discovery of grounded theory: Strategies for qualitative research. Chicago: Aldine Pub.

Helms, J. E., Nicholas, G. \& Green, C. E. (2010). Racism and ethnoviolence as trauma: Enhancing professional training. Traumatology, 16 (4), pp. 53-62. Doi: $10.1177 / 1534765610389595$.

Hernandez-Wolfe, P. (2013). Latinos, A Borderlands View of Latinos, Latin Americans and Decolonization. Rethinking Mental Health. Lanham: Jason Aronson.

Hernandez-Wolfe, P., Acevedo-Velasco, V. E., Victoria, I. \& Volkmann, T. (2015). Transnational Family Therapy Training: A collaborative Learning Experience in Cultural Equity and Humility. Journal of Feminist Family Therapy, 27 (3-4), pp. 134155.Doi:10.1080/08952833.2015.1092813 Published online Nov 17, 2015.

Lincoln, Y. \& Guba, E. (1985). Naturalistic inquiry. Thousand Oaks: Sage.

Maldonado-Torres, N. (2003, marzo). Imperio y colonialidad del ser. Ponencia presentada en el XXIV Congreso Internacional de la Asociación de Estudios Latinoamericanos, Dallas, TX, Estados Unidos.

Mignolo, W. (2000). Diferencia colonial y razón posoccidental. En S. Castro-Gómez (ed.) La reestructuración de las ciencias sociales en América Latina, (pp. 3-28). Bogotá, D. C.: Pensar.

Mignolo, W. (2012). Local Histories/Global designs: Coloniality, subaltern knowledges and border thinking. Princeton: Princeton University Press.

Mir, R. \& Mir, A. (2009). From the colony to the corporation: Studying knowledge transfer across transnational boundaries. Group and Organization Management, 34 (1), pp. 90-113. Doi: 10.1177/1059601108329714.

Padgett, D. K. (1998). Qualitative methods in social work research: Challenges and reviews. Thousand Oaks: Sage Publications. Quijano, A. (2000). Colonialidad del poder, globalización y democracia. Recuperado de: http://www.rrojasdatabank.info/pfpc/ quijan02.pdf 
Segato,R.L.(2003).LasEstructurasElementales de la Violencia: Contrato y Status en la Etiología de la Violencia. Recuperado de: http://www.escuelamagistratura.gov.ar/ images/uploads/estructura_vg-rita_segato. pdf

Victoria-Morales, M.I.(2011).ElEtnocentrismo Jurídico sobre la dimensión intangible de lo sagrado: Una amenaza a la seguridad espiritual de los pueblos ancestrales de la sierra nevada de Santa Marta. Seguridad Humana y Construcción de Paz en Colombia. Barcelona: Huygens.

Victoria-Morales, M. I., Hernandez-Wolfe, P. \& Acevedo-Velasco, V. E. (2013). Colaboraciónyequidad en el entrenamiento de terapeutas familiares comunitarios: diálogo de saberes afrocéntricos y occidentales. Propuesta de investigación aprobada. Documento Interno. Pontificia Universidad javeriana, Cali, Colombia.

Walsh, C. (2006). De-colonialidad e interculturalidad: Reflexiones (des)de proyectos políticos epistémicos. En M. Yapu (comp.) Modernidad y pensamiento descolonizador, (pp. 169-183). La Paz: U-Pieb.

Walsh, C. (2007). Interculturalidad, colonialidad y educación. Revista Educación y Pedagogía, 19 (48), pp. 25-35. 